\title{
REFINEMENTS OF SOME INEQUALITIES RELATED TO JENSEN'S INEQUALITY
}

\author{
YASUO NAKASUJI
}

Abstract. A finite form of Jensen's inequality for a continuous convex function from a topological abelian semigroup to another topological ordered abelian semigroup is given by the author and S.-E. Takahasi. As an application of this abstract Jensen's inequality, two inequalities with respect to geometric mean and arithmetic mean are obtained. The first gives a new refinement of the geometric-arithmetic mean inequality. The second gives a refinement between the arithmetic mean and a certain mean.

Mathematics subject classification (2010): Primary 39B62; Secondary 26B25, 26 A51.

Keywords and phrases: Jensen's inequality, mean, geometric-arithmetic mean inequality, topological abelian semigroup operation.

\section{REFERENCES}

[1] J. Jensen, Sur les fonctions convexes et les inégalités entre les valeurs moyennes, Acta Math 30 (1906), 175-193.

[2] Y. Nakasuji, K. Kumahara And S.-E. TAKahasi, A new interpretation of Jensen's inequality and geometric properties of $\varphi$-means, J. Inequal. Appl., 2011, 2011:48, 15 pp.

[3] Y. NAKASUJ AND S.-E. TAKAHASI, A reconsideration of Jensen's inequality and its applications, J. Inequal. Appl., 2013, 2013:408, 11 pp. 